\title{
Brain Tissue Pulsatility is Increased in Midlife Depression: a Comparative Study Using Ultrasound Tissue Pulsatility Imaging
}

\author{
Thomas Desmidt ${ }^{*, 1,2}$, Bruno Brizard ${ }^{2}$, Paul-Armand Dujardin ${ }^{3}$, Redouane Ternifi ${ }^{2}$, Jean-Pierre Réméniéras ${ }^{2,3}$, \\ Frédéric Patat ${ }^{2,3}$, Frédéric Andersson ${ }^{2}$, Jean-Philippe Cottier ${ }^{2,4}$, Emilie Vierron ${ }^{2,3}$, Valérie Gissot ${ }^{3}$, Kang Kim $^{5}$, \\ Howard Aizenstein ${ }^{6}$, Wissam El-Hage ${ }^{1,2,3}$ and Vincent Camus ${ }^{1,2}$ \\ 'Clinique Psychiatrique Universitaire, CHRU de Tours, Tours, France; '2INSERM U930 Imagerie et Cerveau, Université François-Rabelais de Tours, \\ Tours, France; ${ }^{3}$ NSSERM CIC 1415, Université François-Rabelais de Tours, Tours, France; ${ }^{4}$ Service de Neuroradiologie, CHRU de Tours, Tours, \\ France; ${ }^{5}$ Department of Bioengineering, University of Pittsburgh School of Engineering, Pittsburgh, PA, USA; ${ }^{6}$ Department of Psychiatry, Western \\ Psychiatric Institute and Clinic, University of Pittsburgh School of Medicine, Pittsburgh, PA, USA
}

\begin{abstract}
Cerebrovascular disease (CVD) is consistently associated with late-life depression but poorly documented in midlife depression. It can be hypothesized that the relatively low sensitivity of conventional neuroimaging techniques does not allow the detection of subtle CVD in midlife depression. We used tissue pulsatility imaging (TPI), a novel ultrasound (US) neuroimaging technique that has demonstrated good sensitivity to detect changes in the pulsatility of small brain volumes, to identify early and subtle changes in brain vascular function in midlife depression. We compared the maximum and mean brain tissue pulsatility (MaxBTP and MeanBTP), as identified by TPI, between three groups of middle-aged females matched for age: patients with depression $(n=25)$, patients with remitted depression $(n=24)$ and community controls $(n=25)$. MRI arterial spin labeling, white matter hyperintensities (WMHs) and transcranial doppler (TCD) were used as control conventional markers for CVD. We found no difference in the MRI and TCD measures among the three groups. In contrast, depressive patients showed an increased BTP related to the mean global brain pulsatility (MeanBTP) and no change related to large vessels (MaxBTP) in comparison with the remitted and control groups. US neuroimaging is a highly accurate method to detect brain pulsatility changes related to cerebrovascular functioning, and TPI identified an increased BTP in midlife depressed patients, suggesting early and subtle vascular impairments in this population at risk for CVD such as stroke or WMHs. Because high pulsatility could represent prodromal cerebrovascular changes that damage the brain over time, this paper provides a potential target for blocking the progression of CVD. Neuropsychopharmacology (2017) 42, 2575-2582; doi:I0.1038/npp.2017.1I3; published online 26 July 2017
\end{abstract}

\section{INTRODUCTION}

Cerebrovascular diseases (CVDs) are increasingly recognized as a part of the pathophysiology of late-life depression (LLD). In particular, the vascular depression hypothesis proposed by Alexopoulos et al (1997) has emphasized the role of CVDs in the predisposition, precipitation, or perpetuation of some geriatric depressive syndromes, and recent data suggest that vascular depression is a major health issue that may account for up to half of LLD (Park et al, 2015). Subsequently, Krishnan et al (1997) have proposed a complementary definition of vascular depression based on neuroimaging and have suggested to use MRI as the reference for the characterization of CVDs in LLD. Since then, multiple studies have found a consistent association between LLD and

\footnotetext{
*Correspondence: Dr T Desmidt, Clinique Psychiatrique Universitaire, CHRU de Tours, 2 Boulevard Tonnellé, 37044 Tours Cedex 9, France, Tel: +33 2343789 52, Fax: +332 183708 96,

E-mail: t.desmidt@chu-tours.fr

Received 3 August 2016; revised 20 May 2017; accepted 30 May 20 17; accepted article preview online 6 June 2017
}

MRI markers of CVDs, especially white matter hyperintensities (WMHs) (Herrmann et al, 2008) (Taylor et al, 2013).

In contrast, CVDs are poorly documented in early and midlife depression. However, growing evidence suggests that midlife-depressed patients constitute a group at risk for CVDs. Meta-analyses of epidemiological studies indicate that depression is a risk factor for stroke (Pan et al, 2011) (Dong et al, 2012). Preclinical and clinical studies suggest that depression is associated with pathophysiological mechanisms that may modify vascular function, such as inflammation (Dantzer et al, 2008) or endothelial dysfunction (Isingrini et al, 2009). Taylor et al (2013) recently reviewed the probable mechanisms linking vascular disease with depression and proposed that some underlying vascular processes, involving hypoperfusion, endothelial dysfunction and inflammation, progressively damage the brain and eventually lead to the development of WMHs and depression. However, this suggestion requires more clinical evidence, especially regarding the characterization of the underlying mechanisms linking depression with CVDs. Moreover, and to the best of our knowledge, no study has found signs of CVDs in midlife depression using neuroimaging. 
There may be two reasons for the lack of evidence of CVDs in midlife depression: Either there is no CVDs or only late CVDs in early and midlife depression, or current neuroimaging techniques are not sensitive enough to detect early cerebrovascular impairments. Because WMHs are believed to represent late markers of earlier, progressive and insidious CVDs (Wardlaw et al, 2013), the second assumption may prove to be true. Indeed, based on neuropathological data as well as data showing that the presence of cerebrovascular risk factor in midlife increases the risk of WMHs in late life (Debette et al, 2011), a growing number of authors argue that WMHs are only the tip of the iceberg in terms of damage to the brain (Wardlaw et al, 2013, 2015; Hommet et al, 2011); the pathophysiology could begin years beforehand, with subtle changes in the vasculature that are invisible to conventional imaging and progressively lead to diffuse CVDs (Wardlaw et al, 2013 , 2015; Hommet et al, 2011). However, conventional neuroimaging techniques may misidentify brain CVDs in early and midlife depression, possibly because their limited spatiotemporal resolution does not allow the identification of subtle and early stages of CVDs. Some recent ultrasound (US) neuroimaging techniques may prove to overcome this limitation and be sensitive enough to assess subtle cerebrovascular changes in early and midlife depression.

Indeed, the recent advances in US imaging technology, in both probe technology and signal processing, have made possible the development of so-called tissue pulsatility imaging (TPI). In principle, TPI is similar to transcranial doppler (TCD), except that rather than focusing on only large arteries, it relies on the Echo-B mode of a modern US scanner to measure the pulsatile movements of large brain regions, which allow a very high spatiotemporal level of detection (micrometers/millisecond) of brain volume changes related to pulsatile cerebral blood flow (CBF). Periodic changes in blood volume cause the brain to expand and relax over the cardiac cycle, and TPI measures the pulsatile signals from thousands of sample volumes in an US image plane, consistently with the principle of plethysmography (Kucewicz et al, 2004). TPI has been validated on phantoms (Kucewicz et al, 2004) and on healthy volunteers. It was shown that BTP was increased during visual stimulation (Kucewicz et al, 2007) and was decreased during hyperventilation (Kucewicz et al, 2008), which suggests that BTP is closely related to CBF changes and cerebrovascular reactivity (CVR), consistently with the notion that pulsatile $\mathrm{CBF}$ and cerebrovascular functioning are major determinants of brain pulsatility (Wagshul et al, 2011). Our team also found that TPI was informative in clinical settings, as BTP was shown to be inversely correlated with WMH load (Ternifi et al, 2014). In another pilot study (Desmidt et al, 2011), we investigated the use of TPI in LLD, and we found that BTP was markedly lower in diabetic depressed patients compared with diabetic non-depressed subjects. Thus, TPI has been shown to identify changes in the BTP of patients with constituted CVDs, but to date, we lack data on the use of TPI on patients at risk of CVDs-including early and midlife depressed patients-who may exhibit early and subtle cerebrovascular changes.

The goal of our study was to characterize the BTP of midlife depressed females with TPI. Based on previous results on LLD and CVDs, we hypothesized that BTP would be lower in depressed patients compared with controls and remitted subjects. We also compared BTP with WMHs and CBF in the middle cerebral artery (MCA), as assessed by TCD and MRI arterial spin labeling (ASL), as potential cofounders of BTP (Ternifi et al, 2014).

\section{MATERIALS AND METHODS}

\section{Participants}

Seventy-five females aged 18-55 years were recruited and divided into three equal groups as part of the EMPHILINE project (NCT02026622 on clinicaltrials.gov), the principal objective of which was to investigate the cardiovascular and CVR in depression during emotional tasks; the baseline data are reported in the present article. To reduce variability, only females were recruited because previous findings have found significant differences in cerebrovascular properties between age-matched males and females (Parkes et al, 2004). Subjects in the depressive group (group D; $n=25$ ) were inpatients or outpatients from the psychiatric inpatient units of the University Hospital of Tours, France. A psychiatrist (T.D.) diagnosed the patients as depressed according to the DSMIV criteria for major depressive disorder (MDD) and a MADRS score higher than 21. The medical records of the psychiatric inpatient units were used to screen subjects for the remission group (group $\mathrm{R} ; n=25$ ). Subjects in the $\mathrm{R}$ group had to have at least one documented history of MMD in the past ten years but no criteria for current MDD in the last 6 months, and they needed a MADRS score lower than 9 . Subjects in the control group (group C; $n=25$ ) were recruited from the local community and from the records of the research center of the Hospital of Tours, France. They had to have no history of MDD or any psychiatric disorder and have a MADRS score lower than 9. The three groups were matched for age. Non-inclusion criteria for all subjects were: (1) any history of psychotic, bipolar, or substanceabuse disorders or suspicion of severe cognitive impairment (MMSE <25), (2) any history of severe CVDs (myocardial infarction, arrhythmia, etc.) or neurological disorders (stroke, brain tumor, severe concussion, migraine, etc.), (3) any current instable medical condition, (4) current use of beta blockers or antipsychotics, (5) smoking over 10 pack-years, (6) auditory or visual impairments, (7) pregnancy or no reliable contraception, (8) contraindication for MRI, and (9) legal guardianship. The exclusion criterion was having no temporal window because US are attenuated by the thickness of the skull. Informed consent was obtained from all subjects, and the study protocol was approved by the local human ethical committee.

\section{Clinical Assessments}

Either a psychiatrist (T.D.) or a trained medical doctor (V.G.) from the research center performed the clinical and psychometric assessments in the research center of the University Hospital of Tours, France. Medical history and medication intake were recorded. The clinical assessment included blood pressure (measured twice, at rest and immediately before the US assessment), height and weight measurements. Psychometric assessments included the Structured Clinical Interview for DSM-IV (SCID) for current 
and lifetime MDD, the MADRS for severity of depression and the Mini Mental Status Examination (MMSE) for subjects over 50 to assess global cognitive functioning and screening for dementia.

\section{Magnetic Resonance Imaging Protocol}

MRIs were performed immediately after the psychometric assessment (except for five subjects for whom an MRI session was performed 1 week later owing to MRI unavailability) on a 3-Tesla Siemens Verio scanner (Siemens AG, Erlangen, Germany). T2-FLAIR sequence (fluid-attenuated inversion recuperation), ASL, and high-resolution T1weighted MRI 3D volumes sequence (192 contiguous sagittal slices; $1 \mathrm{~mm}$ slice thickness; $\mathrm{RT}=1.9 \mathrm{~s} ; \mathrm{TE}=2.42 \mathrm{~ms}$; $\mathrm{TI}=0.9 \mathrm{~ms} ; \mathrm{FA}=9^{\circ}$; in-plane resolution: $1 \times 1 \mathrm{~mm}$ ) were acquired for each subject.

\section{WMHs Assessment}

The WMH analysis method was based on the one described by Gurol et al (2006), using a FLAIR sequence (70 contiguous axial slices; $5 \mathrm{~mm}$ slice thickness; $\mathrm{RT}=9 \mathrm{~s}$; $\mathrm{TE}=95 \mathrm{~ms}$; $\mathrm{TI}=2.5 \mathrm{~s}$; in-plane resolution: $1 \times 1 \mathrm{~mm}$ ). This method includes a visual and volumetric analysis, based on the fluid-attenuated inversion recuperation sequence. Three ROIs were located manually in a healthy WM ROI for each subject to obtain the mean intensity (mWH) and standard deviation (sdWM) of the T2-FLAIR value of the white matter tissue. Then, on each section, large and coarse ROI were drawn in areas that may contain lesions. Thresholding was then performed in the latter large ROI, using $\mathrm{mWH}$ $+\left(3^{*} \mathrm{~d} d W M\right)$ as a threshold value. A visual analysis of each section allowed the elimination of the gaps' volume. The $\mathrm{WMH}$ volume was normalized by the total intracranial volume to correct for variations in head size.

\section{ASL-MRI Assessment}

Cerebral blood flow maps (relCBF) were computed from pASL sequences (45 pairs of label/control ASL images; 17 contiguous axial slices; $5 \mathrm{~mm}$ slice thickness; $\mathrm{RT}=2.6282 \mathrm{~s}$; $\mathrm{TE}=13 \mathrm{~ms} ; \mathrm{FA}=90^{\circ} ; \mathrm{TI} 1=0.7 \mathrm{~s} ; \mathrm{TI} 2=1.8 \mathrm{~s}$; in-plane resolution: $4 \times 4 \mathrm{~mm}$; enabled prospective motion correction). For each subject, the CBF mean value was extracted from an ROI corresponding to MCA territory. Each individual MCA ROI was computed using the 3D-T1 image and based on Duvernoy's work (Anon, 2009) and Destrieux's atlas (Destrieux et al, 2010). A transformation matrix corresponding to the registration of ASL images to the structural 3D-T1 image was used to register each individual MCA ROI to each relCBF map.

\section{US Protocol}

US protocol was performed immediately after MRI sessions (except for five subjects who had their MRI session rescheduled; they had a US immediately after the clinical assessment) on an Antares medical scanner (Siemens Healthcare, Germany) by a single biophysics technician (B.B.), trained for TCD, who was blinded to the MRI results and the depressive status. Transcranial acquisitions were performed with a PX4-1 phased-array transducer (Siemens Healthcare; $1.82 \mathrm{MHz}$ emission frequency, $70^{\circ}$ field of view, $112 \times 3$ elements $(1.5 \mathrm{D}))$. Measurements were performed through the right temporal bone window, which is the thinnest zone of the skull, with the probe positioned perpendicularly to the skull and maintained by a mechanical holder to reduce artifacts caused by the operator's and the subject's movements. Subjects were asked to remain in the supine position, try not to move, close their eyes and breathe normally.

The US scanner was first switched on Doppler mode for TCD acquisition. Color doppler was used to center the US beam on the right MCA. The pulsatility index (PI-ratio of the difference between maximal and minimal velocities over mean velocity) and the maximum and mean velocities of the right MCA were acquired and automatically computed by the scanner from a $10 \mathrm{~s}$ doppler sequence.

The US scanner was then switched to Echo-B mode to perform TPI measurements centered on the MCA. To limit US attenuation, we adjusted the depth of acquisition between 3 and $9 \mathrm{~cm}$. With this configuration, we explored the circle of Willis and a transversal slice of the temporal hemispheres.

For each subject, the protocol consisted of four acquisitions of $10 \mathrm{~s}$ repeated every $26 \mathrm{~s}$ with an acquisition frame rate of 30 images/s (total of 297 frames). The US scanner provided direct access to beam-formed radiofrequency (RF) lines, which were used to estimate BTP. The data were then downloaded for offline analysis with MATLAB software (MathWorks, USA).

Our method described in the following lines assesses the axial component of brain displacements. Because the US beam is directed toward the center of the brain, BTP measures primarily relate to volume variations. Axial BTP was estimated using a $1 \mathrm{D}$-intercorrelation method between successive acquisition frames. The maximum of the normalized intercorrelation coefficient, Txy/sqrt(TxxTyy), was used to estimate the delay between successive kernels on RF lines at a specific depth. The normalized correlation coefficient had to be higher than 0.7 to validate the measurement. A kernel size of eight periods (176 points, 4 wavelengths, $3.3 \mathrm{~mm}$ at $1.82 \mathrm{MHz}$ ) was chosen. Parabolic interpolation was performed around the three maximum points of the $1 \mathrm{D}$ correlation to improve the precision of the displacement measurement. With the signal to noise ratio obtained after propagation through the skull, the noise level of BTP was $0.8 \mu \mathrm{m}$ (computed based on the Cramer-Rao lower bound formula, previously described for US measure of displacement in (Byram et al, 2013)). For a better spatial discretization along RF lines, an overlap rate of $80 \%$ of the kernel was computed, resulting in a $660 \mu \mathrm{m}$ spatial discretization. A bandpass filter (low cutoff $=0.75 \mathrm{~Hz}$; high cutoff $=5.0 \mathrm{~Hz}$ ) was then applied to focus on displacements related to heart rate and to filter out slow and fast movements due to, respectively, respiration and artifacts.

We obtained a 3D matrix of BTP: axial displacements along the $z$ axis (RF lines), $x$ axis (along the 112 elements of the probe) and time. The temporal evolution of BTP was analyzed at each position of the 2D plane (curves of the displacement as a function of time). We used two criteria to filter out artifacts and focus on physiological signals, supposed to be stable in terms of periodicity and amplitude. The first criterion was used to investigate the periodicity of 
Table I Demographic and Clinical Characteristics of the Population

\begin{tabular}{|c|c|c|c|c|}
\hline & $\begin{array}{c}\text { Depressed } \\
\text { patients } \\
(n=25)\end{array}$ & $\begin{array}{c}\text { Remitted } \\
\text { patients } \\
(n=24)\end{array}$ & $\begin{array}{l}\text { Controls } \\
(n=25)\end{array}$ & $p$-value \\
\hline Age (years) & $41.0(12.6)$ & $42.9(11.6)$ & $42.7(13)$ & 0.958 \\
\hline \multicolumn{5}{|l|}{$\begin{array}{l}\text { Cardiovascular risk } \\
\text { factors }\end{array}$} \\
\hline Smoke & || (44\%) & $10(42 \%)$ & $5(20 \%)$ & 0.148 \\
\hline $\mathrm{BMI}\left(\mathrm{kg} / \mathrm{m}^{2}\right)$ & $22.3(5.3)$ & $23.5(5.1)$ & $22.2(4.3)$ & 0.430 \\
\hline $\begin{array}{l}\text { High blood } \\
\text { pressure }\end{array}$ & $2(8 \%)$ & $3(12 \%)$ & $2(8 \%)$ & 0.788 \\
\hline High cholesterol & $2(8 \%)$ & 0 & I (4\%) & 0.767 \\
\hline Diabetes & 0 & 0 & 0 & - \\
\hline $\begin{array}{l}\text { Baseline SBP } \\
(\mathrm{mmHg})\end{array}$ & $122(18)$ & $125(17.5)$ & $117(18)$ & 0.180 \\
\hline $\begin{array}{l}\text { Baseline DBP } \\
(\mathrm{mmHg})\end{array}$ & $73(14)$ & $77(12)$ & $72(13)$ & 0.245 \\
\hline \multicolumn{5}{|l|}{ Medication intake } \\
\hline Antidepressant & II (44\%) & $10(42 \%)$ & 0 & 0.0004 \\
\hline Anxiolytic & 7 (28\%) & $4(17 \%)$ & | (4\%) & 0.053 \\
\hline Mood stabilizer & $3(12 \%)$ & $2(8 \%)$ & 0 & 0.193 \\
\hline Antihypertensive & I (4\%) & | (4\%) & I (4\%) & I \\
\hline \multicolumn{5}{|l|}{$\begin{array}{l}\text { Depression } \\
\text { characteristics }\end{array}$} \\
\hline MADRS & $33(8)$ & $4(4)$ & $0(2)$ & $<10^{-4}$ \\
\hline $\begin{array}{l}\text { Age at onset } \\
\text { (years old) }\end{array}$ & $25.5(15.25)$ & $25.5(9.25)$ & - & 0.742 \\
\hline $\begin{array}{l}\text { Number of } \\
\text { episodes }\end{array}$ & I (I) & $2(1)$ & - & 0.596 \\
\hline
\end{tabular}

Abbreviations: BMI: body mass index; DBP: diastolic blood pressure; MADRS: Montgomery and Asberg Depression Rating Scale; SBP: systolic blood pressure. Values are expressed as median (Interquartile Range) or $n$ (\%). Comparisons were performed with Kruskal-Wallis tests (quantitative data) and chi-squared tests (qualitative data). Blood pressure was measured immediately before the ultrasound assessment.

the brain pulsations and consisted in the ratio of the second maximum peak over the central peak (SMP/CP) of the temporal autocorrelation function of each kernel. If the ratio SMP/CP was higher than 0.6 , the record was validated and confronted to the second criterion. This threshold of 0.6 was chosen based on previous studies (Desmidt et al, 2011, Ternifi et al, 2014, Biogeau et al, in press) and the empirical observations of several TPI signals. The second criterion was used to investigate the difference in amplitudes between pulsations in each curve. The cumulated standard deviation (CSTD) of the pulsations was calculated to inform on the data dispersion and was normalized to the peak-to-peak amplitude (Umean) of the mean temporal curve. If the ratio CSTD/Umean was lower than 0.25 , the record was validated, otherwise rejected. This threshold of 0.25 was chosen based on the same reasons of the first criterion.

Finally, the medians (interquartile range) of the criteria showed similar values among groups, with no significant
Table 2 Comparisons of MRI, TCD, and TPI Measures Among the Three Groups

\begin{tabular}{|c|c|c|c|c|}
\hline & $\begin{array}{c}\text { Depressed } \\
\text { patients } \\
(n=25)\end{array}$ & $\begin{array}{c}\text { Remitted } \\
\text { patients } \\
(n=24)\end{array}$ & $\begin{array}{l}\text { Controls } \\
(n=25)\end{array}$ & $p$-value \\
\hline \multicolumn{5}{|l|}{ MRI } \\
\hline $\begin{array}{l}\text { Total WMH } \\
\left(\mathrm{cm}^{3}\right)\end{array}$ & $0.82(0.83)$ & $0.63(0.67)$ & $0.70(0.98)$ & 0.167 \\
\hline $\begin{array}{l}\text { Right hemisphere } \\
\text { WMH }\left(\mathrm{cm}^{3}\right)\end{array}$ & $0.41(0.44)$ & $0.32(0.31)$ & $0.31(0.36)$ & 0.480 \\
\hline $\begin{array}{l}\text { Right MCA ASL } \\
\text { (arbitrary unit) }\end{array}$ & $105(53)$ & $106(4 \mid .5)$ & I I 0.5 (27.25) & 0.450 \\
\hline \multicolumn{5}{|l|}{$T C D$} \\
\hline $\begin{array}{l}\text { MCA max } \\
\text { velocity }(\mathrm{m} / \mathrm{s})\end{array}$ & 80.9 (39.7) & $100.4(21.7)$ & $93.7(34.9)$ & 0.100 \\
\hline $\begin{array}{l}\text { MCA mean } \\
\text { velocity }(\mathrm{m} / \mathrm{s})\end{array}$ & $49.9(25.5)$ & $65.1(20.5)$ & $56.1(26.1)$ & 0.131 \\
\hline MCA PI & $0.88(0.31)$ & $0.88(0.27)$ & $0.90(0.24)$ & 0.956 \\
\hline \multicolumn{5}{|l|}{ TPI } \\
\hline $\begin{array}{l}\text { MaxBTP PtoP } \\
(\mu \mathrm{m})\end{array}$ & I I 5.8 (53.7) & $97.5(35.1)$ & $106.4(50.1)$ & 0.439 \\
\hline $\begin{array}{l}\text { MaxBTP RMS } \\
(\mu \mathrm{m})\end{array}$ & $36.1(17.9)$ & $31.2(12.1)$ & $34.1(17.5)$ & 0.484 \\
\hline $\begin{array}{l}\text { MeanBTP PtoP } \\
(\mu \mathrm{m})\end{array}$ & $24.6(10)$ & 20.1 (9.3) & $20.0(8.7)$ & 0.046 \\
\hline $\begin{array}{l}\text { MeanBTP RMS } \\
(\mu \mathrm{m})\end{array}$ & $8.6(5.3)$ & $6.7(3.7)$ & $7.0(3.4)$ & 0.031 \\
\hline
\end{tabular}

Abbreviations: ASL, arterial spin labeling; BTP, brain tissue pulsatility; MCA, middle cerebral artery; MRI, magnetic resonance imaging; PI, pulsatility index; PtoP, peak-to-peak; RMS, root mean square; TCD, transcranial Doppler; TPI, tissue pulsatility imaging; WMH, white matter hyperintensities.

Values are expressed as the median (interquartile range). Comparisons were performed with Kruskal-Wallis tests.

difference (for criterion 1: $\mathrm{M}=0.75(0.19), \mathrm{M}=0.78(0.13)$, $0.79(0.16)$, for group $\mathrm{D}, \mathrm{R}$, and $\mathrm{C}$ respectively, $p=0.993$; for criterion 2: $\mathrm{M}=0.21(0.17), \mathrm{M}=0.19(0.10), \mathrm{M}=0.24(0.20)$, for group $\mathrm{D}, \mathrm{R}$, and $\mathrm{C}$ respectively, $p=0.572$ ), and suggested a significant stability in periodicity and amplitude.

From this final matrix, we isolated two curves, MaxBTP and MeanBTP, corresponding, respectively, to the curve with the maximum of the mean peak-to-peak amplitude (averaged between cycles) and to the averaging of all curves in the matrix. MaxBTP and MeanBTP thus correspond to the pulsatility of, respectively, the largest artery (ie, MCA) and the mean pulsatility in the whole region of acquisition. Finally, we calculated two parameters for each curve -the average peak-to-peak amplitude (BTP_PtoP) and the root mean square (BTP_RMS) - which reflect the largest amplitude and the displacement power value, respectively.

\section{Statistical Analyses}

Shapiro-Wilk tests determined that the variables were not normally distributed. We therefore performed non- 
parametric analyses for group comparisons (Kruskal-Wallis with Dunn's post-test).

\section{RESULTS}

\section{Clinical Characteristics}

One patient in group $\mathrm{R}$ was excluded for having no temporal window. As shown in Table 1, there was no difference in age, cardiovascular risk factors or baseline blood pressure among the three groups. Antidepressant intake was significantly higher in groups $\mathrm{D}$ and $\mathrm{R}$ compared with group $\mathrm{C}$ $\left(p<10^{-4}\right)$, but there was no difference between groups D and R $(p=0.857)$. The MADRS score was significantly greater in group D compared with group $\mathrm{R}\left(p<10^{-4}\right)$, in group D compared with group $\mathrm{C} p\left(<10^{-4}\right)$ and in group $\mathrm{R}$ compared with group $\mathrm{C}(p=0.011)$.

\section{MRI and US Measures}

As shown in Table 2, all subjects except 2 (one in group D and one in group C, who had, respectively, 15.8 and $19.4 \mathrm{~cm}^{3}$ total WMHs) showed very low WMHs, and there was no difference in the total or right hemisphere WMHs among the three groups. Similarly, there was no difference in MCA ASL $\mathrm{CBF}$ or in the TCD measures among the three groups. In contrast, as shown in Figure 1, TPI measures showed a higher MeanBTP for both Peak-to-Peak and RMS values in group D compared with the two other groups, whereas we found no difference in the MaxBTP values. Finally, Figure 2 and Supplementary Video 1 show BTP color maps of three representative subjects in each group and provide visual examples of higher global brain pulsatility in depressed patients.

\section{DISCUSSION}

We found that MeanBTP was significantly higher in depressed subjects compared with controls and remitted subjects, whereas we found no difference in MCA CBF, as assessed by ASL, TCD, and TPI. MeanBTP corresponds to the averaged value of thousands of brain volume pulsations and informs on the mean global brain pulsatility related to both large and small arteries. In contrast, MaxBTP informs on only the largest pulsatility, which is likely due to MCA. Thus, our results suggest that midlife depression can be characterized by high global brain pulsatility without significant changes in large-artery CBF.

Growing evidence from the literature suggests that excessive intracranial pulsatility correlates with CVDs and brain atrophy. A recent study (Wåhlin et al, 2014) that used MRI and invasive methods of pulsatility measurement on healthy volunteers found that higher intracranial cardiacrelated pulsatility was associated with lower brain volume, especially in the hippocampus and temporal lobe, suggesting that excessive pulsatility correlates with atrophy. Similarly, other studies found that high pulsatility was observed in various CVDs, including WMHs (Tsao et al, 2013) and
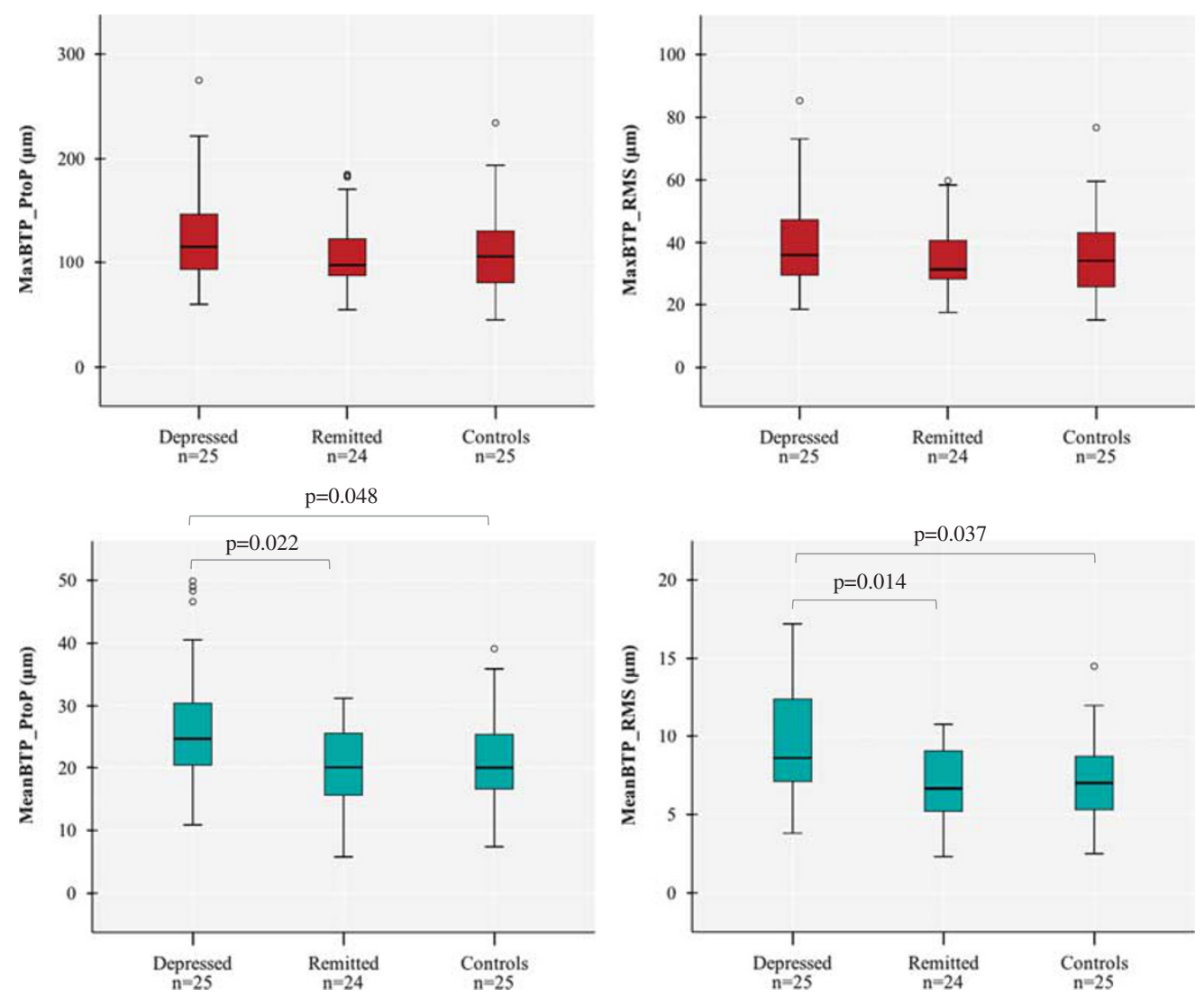

Figure I Comparisons of the TPI measures of MaxBTP (in red-top left: Peak-to-Peak, top right: RMS) and MeanBTP (in blue - bottom left: Peak-to-Peak, bottom right: RMS) among the three groups. 

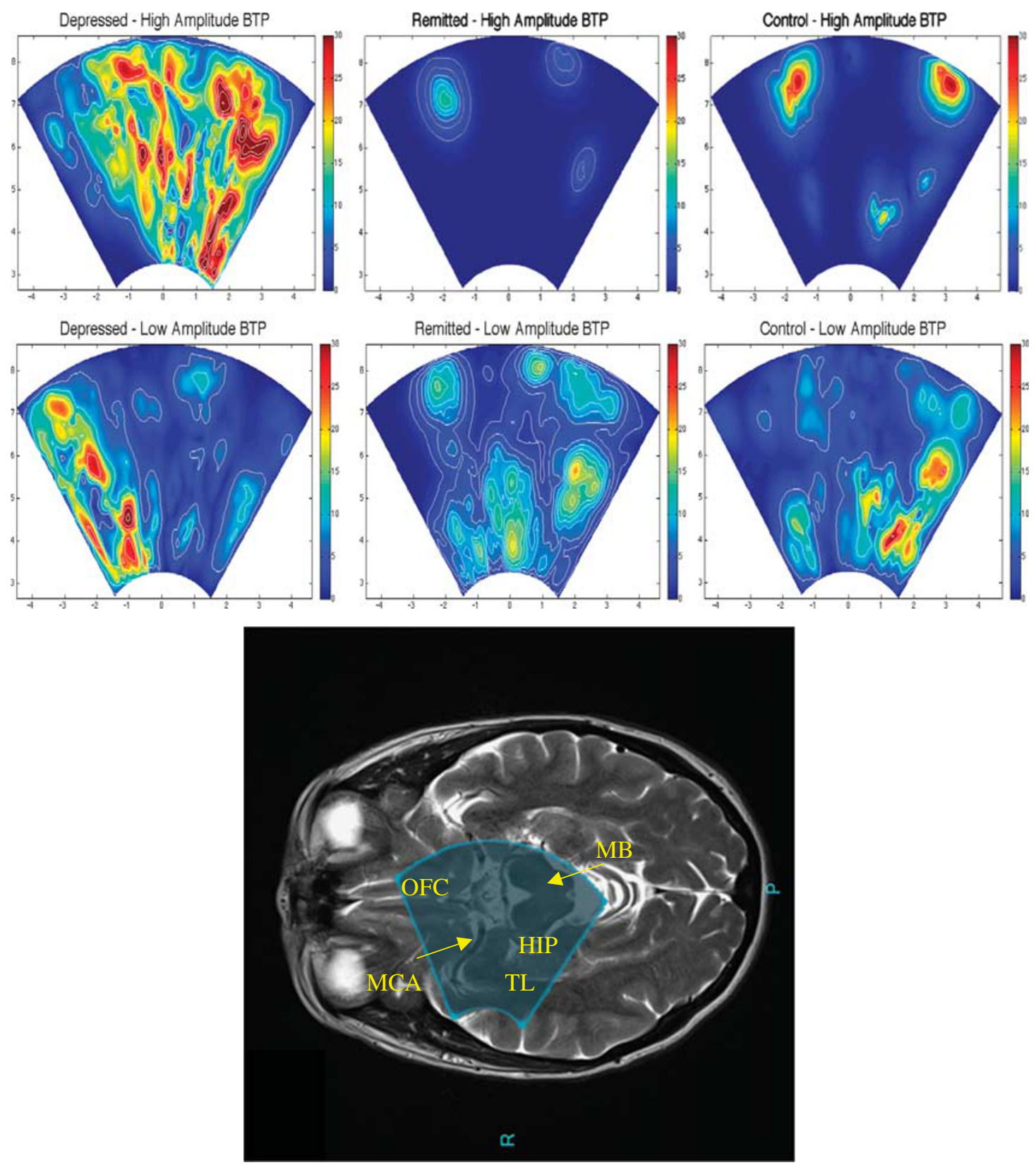

Figure 2 Brain tissue pulsatility (BTP) color maps from three representative subjects of each group and picture of the ultrasound beam of the TPI overlaid on a MRI axial image. We filtered BTP matrixes with a threshold equal to $50 \%$ of the MaxBTP of the depressed subject to distinguish between high- and lowamplitude BTP, shown, respectively, at the top and bottom of the figure. We also applied the Horn-Schunck method to estimate the optical flow and displacement speed of each volume in the region of acquisition, the color maps of which show maximum values. Depressed subjects (left) exhibit more distributed high BTP compared with remitted (center) and control (right) subjects, who, in contrast, exhibit very localized high BTP, probably around the main large arteries of the circle of Willis. Low BTP appears to be similarly distributed in each of the three subjects in various small regions, possibly corresponding to small vessels. These maps suggest that high brain pulsatility, normally limited to large vessels in remitted and control subjects, affects the whole brain in depressed patients, consistently with our result of a higher MeanBTP in depressed patients. As shown on the bottom figure, the ultrasound beam of the TPI overlays parts of the temporal lobes (including parts of the hippocampus), the orbitofrontal cortex and the brainstem (midbrain). Hip: hippocampus; MB: midbrain; MCA: middle cerebral artery; OFC: orbitofrontal cortex; TL: temporal lobe.

cognitive disorders (Sabayan et al, 2012) (Tsao et al, 2013) (Isingrini et al, 2009). However, these studies used TCD, which inform on only large-vessel pulsatility. In contrast, TPI provides direct monitoring of global brain pulsatility related to both large and small vessels, and, to our knowledge, our study is the first to show direct evidence of increased global pulsatility in midlife depressed patients, a group at risk for CVDs.
The observation that increased pulsatility is state dependent is consistent with findings that WMH progression is observed in individuals who remain depressed and do not respond to antidepressants (Khalaf et al, 2015), suggesting that the longer one stays depressed, the worse their cerebrovascular health. Because high pulsatility could represent prodromal cerebrovascular changes that damage the brain over time and potentiate or mediate the 
progression of CVD such as WMHs, this paper provides a potential target for blocking the progression of CVD.

The result of an increased BTP in midlife depression is in contradiction with our a priori hypothesis. Nevertheless, this is the first study to investigate BTP with TPI in midlife depression and while there is a vast literature on CVDs in LLD, little is known about the cerebrovascular functioning in midlife depression. In a previous pilot study (Desmidt et al, 2011), we found that BTP was decreased in LLD patients with long-term diabetes. In this pilot study, we did not control for WMHs and elderly diabetic patients are likely to exhibit high level of WMHs. As WMHs are strongly and inversely correlated with BTP (Ternifi et al, 2014), the decreased BTP in LLD may indicate a larger WMHs volume in depressed patients, which is consistent with numerous studies that found a constant greater volume of WMHs in elderly depressed subjects compared with controls (Taylor et al, 2013). In contrast, WMHs are rarely observed in midlife depression. WMHs consist in significant and irreversible structural changes that dramatically reduce BTP. However, recent findings suggest a pre-stage to WMHs that involves functional rather than structural changes, including excessive brain pulsatility (O’Rourke and Hashimoto, 2007).

Taken together, our results suggest that there may be two profiles of BTP in depression, depending on the patient's age and the presence of CVDs: low BTP in LLD patients with WMHs and high BTP in midlife depressed patients without WMHs. A study comparing BTP in midlife vs LLD, with WMHs as covariate, is required to confirm this suggestion. In addition, other studies involving depressed males would be required to investigate a possible gender effect of BTP on depression, as we recruited only females in our present study. Finally, longitudinal studies investigating the link between high BTP and the development of WMHs are required.

TPI has some limitations, including the inability to measure the whole brain in one acquisition, the inability to locate brain structures and limitations due to bone attenuation. However, it is an easy-to-realize, costless and noninvasive technique that could ultimately provide a sophisticated office tool for clinicians to detect subtle impairments in the cerebrovascular functioning of patients at risk for CVDs, especially depressive patients, to guide treatment and prevention strategies. We also foresee multiple perspectives for the development of TPI, including its coupling with MRI neuronavigation to allow anatomical localization, its use in preclinical settings to investigate molecular physiology that influences BTP, its implementation in large and longitudinal studies to assess BTP changes with treatment and symptoms remission in depression, and the development of sophisticated signal processing, such as frequency domain analyses.

\section{FUNDING AND DISCLOSURE}

The authors declare no conflict of interest.

\section{ACKNOWLEDGMENTS}

This study received grant from the French National Research Agency (Agence Nationale de la Recherche: ANR-2011-
EMCO-005-'Emphiline project') and the Planiol Foundation for brain study (Fondation Planiol pour le Cerveau), Varennes, France.

\section{REFERENCES}

Alexopoulos GS, Meyers BS, Young RC, Campbell S, Silbersweig D, Charlson M (1997). "Vascular depression" hypothesis. Arch Gen Psychiatry 54: 915-922.

Anon (2009). Internal Architecture of the Brain Stem with Key Axial Section. In: Duvernoy's Atlas of the Human Brain Stem and Cerebellum. Springer: Vienna. pp 53-93.

Biogeau J, Desmidt T, Dujardin P-A, Ternifi R, Eudo C, Vierron E et al (In Press). Ultrasound tissue pulsatility imaging suggests impairment in global brain pulsatility and small vessels in elderly patients with orthostatic hypotension. J Stroke Cerebrovasc Dis 26: 246-251.

Byram B, Trahey GE, Palmeri M (2013). Bayesian speckle tracking. Part II: biased ultrasound displacement estimation. IEEE Trans Ultrason Ferroelectr Freq Control 60: 144-157.

Dantzer R, O'Connor JC, Freund GG, Johnson RW, Kelley KW (2008). From inflammation to sickness and depression: when the immune system subjugates the brain. Nat Rev Neurosci 9: 46-56.

Debette S, Seshadri S, Beiser A, Au R, Himali JJ, Palumbo C et al (2011). Midlife vascular risk factor exposure accelerates structural brain aging and cognitive decline. Neurology 77: 461-468.

Desmidt T, Hachemi ME, Remenieras J-P, Lecomte P, FerreiraMaldent N, Patat F et al (2011). Ultrasound brain tissue pulsatility is decreased in middle aged and elderly type 2 diabetic patients with depression. Psychiatry Res 193: 63-64.

Destrieux C, Fischl B, Dale A, Halgren E (2010). Automatic parcellation of human cortical gyri and sulci using standard anatomical nomenclature. NeuroImage 53: 1-15.

Dong J-Y, Zhang Y-H, Tong J, Qin L-Q (2012). Depression and risk of stroke: a meta-analysis of prospective studies. Stroke J Cereb Circ 43: 32-37.

Gurol ME, Irizarry MC, Smith EE, Raju S, Diaz-Arrastia R, Bottiglieri $T$ et al (2006). Plasma beta-amyloid and white matter lesions in $A D$, MCI, and cerebral amyloid angiopathy. Neurology 66: 23-29.

Herrmann LL, Le Masurier M, Ebmeier KP (2008). White matter hyperintensities in late life depression: a systematic review. J Neurol Neurosurg Psychiatry 79: 619-624.

Hommet C, Mondon K, Constans T, Beaufils E, Desmidt T, Camus V et al (2011). Review of cerebral microangiopathy and Alzheimer's disease: relation between white matter hyperintensities and microbleeds. Dement Geriatr Cogn Disord 32: 367-378.

Isingrini E, Desmidt T, Belzung C, Camus V (2009). Endothelial dysfunction: a potential therapeutic target for geriatric depression and brain amyloid deposition in Alzheimer's disease? Curr Opin Investig Drugs Lond Engl 2000 10: 46-55.

Khalaf A, Edelman K, Tudorascu D, Andreescu C, Reynolds CF, Aizenstein H (2015). White matter hyperintensity accumulation during treatment of late-life depression. Neuropsychopharmacology 40: 3027-3035.

Krishnan KRR, Hays JC, Blazer DG (1997). MRI-defined vascular depression. Am J Psychiatry 154: 497-500.

Kucewicz JC, Dunmire B, Giardino ND, Leotta DF, Paun M, Dager SR et al (2008). Tissue pulsatility imaging of cerebral vasoreactivity during hyperventilation. Ultrasound Med Biol 34: 1200-1208.

Kucewicz JC, Dunmire B, Leotta DF, Panagiotides H, Paun M, Beach KW (2007). Functional tissue pulsatility imaging of the brain during visual stimulation. Ultrasound Med Biol 33: 681-690.

Kucewicz JC, Huang L, Beach KW (2004). Plethysmographic arterial waveform strain discrimination by Fisher's method. Ultrasound Med Biol 30: 773-782. 
O’Rourke MF, Hashimoto J (2007). Mechanical factors in arterial aging: a clinical perspective. J Am Coll Cardiol 50: 1-13.

Pan A, Sun Q, Okereke OI, Rexrode KM, Hu FB (2011). Depression and risk of stroke morbidity and mortality: a meta-analysis and systematic review. JAMA 306: 1241-1249.

Parkes LM, Rashid W, Chard DT, Tofts PS (2004). Normal cerebral perfusion measurements using arterial spin labeling: reproducibility, stability, and age and gender effects. Magn Reson Med 51: 736-743.

Park JH, Lee SB, Lee JJ, Yoon JC, Han JW, Kim TH et al (2015). Epidemiology of MRI-defined vascular depression: a longitudinal, community-based study in Korean elders. J Affect Disord 180: 200-206.

Sabayan B, Jansen S, Oleksik AM, van Osch MJP, van Buchem MA, van Vliet $\mathrm{P}$ et al (2012). Cerebrovascular hemodynamics in Alzheimer's disease and vascular dementia: a meta-analysis of transcranial Doppler studies. Ageing Res Rev 11: 271-277.

Taylor WD, Aizenstein HJ, Alexopoulos GS (2013). The vascular depression hypothesis: mechanisms linking vascular disease with depression. Mol Psychiatry 18: 963-974.
Ternifi R, Cazals X, Desmidt T, Andersson F, Camus V, Cottier J-P et al (2014). Ultrasound measurements of brain tissue pulsatility correlate with the volume of MRI white-matter hyperintensity. J Cereb Blood Flow Metab 34: 942-944.

Tsao CW, Seshadri S, Beiser AS, Westwood AJ, DeCarli C, Au R et al (2013). Relations of arterial stiffness and endothelial function to brain aging in the community. Neurology 81: 984-991.

Wagshul ME, Eide PK, Madsen JR (2011). The pulsating brain: a review of experimental and clinical studies of intracranial pulsatility. Fluids Barriers CNS 8: 5.

Wåhlin A, Ambarki K, Birgander R, Malm J, Eklund A (2014). Intracranial pulsatility is associated with regional brain volume in elderly individuals. Neurobiol Aging 35: 365-372.

Wardlaw JM, Valdés Hernández MC, Muñoz-Maniega S (2015). What are white matter hyperintensities made of? Relevance to vascular cognitive impairment. J Am Heart Assoc 4: 001140.

Wardlaw J, Smith C, Dichgans M (2013). Mechanisms underlying sporadic cerebral small vessel disease: insights from neuroimaging. Lancet Neurol 12: 483-497.

Supplementary Information accompanies the paper on the Neuropsychopharmacology website (http://www.nature.com/npp) 